\title{
Associação entre os escores da escala Sequential Organ Failure Assessment e a mortalidade de pacientes sob ventilação mecânica internados em uma Unidade de Terapia Intensiva
}

\author{
Association between Sequential Organ Failure Assessment scores and mortality in mechanically \\ ventilated patients admitted to an Intensive Care Unit
}

Asociación entre puntuaciones de la escala Sequential Organ Failure Assessment y la mortalidad de pacientes con ventilación mecánica ingresados en una Unidad de Cuidados Intensivos

\section{Resumo}

A sepse é uma síndrome definida como uma resposta sistêmica e desordenada a determinado quadro infeccioso, causada por qualquer microrganismo, apresentando-se em diferentes estágios clínicos de um mesmo processo fisiopatológico. É fundamental a identificação precoce da sepse, além da utilização de escalas para identificar rapidamente os órgãos acometidos a fim de otimizar a administração de determinados recursos hospitalares. O objetivo deste estudo é investigar a associação entre os escores da escala Sequential Organ Failure Assessment (SOFA) e a mortalidade de pacientes internados em uma unidade de terapia intensiva. Trata-se de um estudo de coorte prospectivo, com análise documental. O sistema respiratório e o de coagulação não apresentaram associação com a mortalidade. Entretanto, os escores dos outros 4 sistemas (hepático, cardiovascular, neurológico e renal) apresentaram forte associação com a mortalidade na amostra de estudo (p-valor <0,05). Foi possível verificar que a escala de SOFA é um instrumento preditor da mortalidade associada à disfunção orgânica em pacientes com infeção, internados em uma unidade de terapia intensiva.

Palavras-chave: Sepse; Escores de disfunção orgânica; Escala SOFA; Unidades de terapia intensiva.

\begin{abstract}
Sepsis is a syndrome defined as a systemic and disordered response to a specific infectious condition, caused by any microorganism, presenting itself in different clinical stages of the same pathophysiological process. Early identification of sepsis is essential, in addition to the use of scales to quickly identify the affected organs in order to optimize the administration of certain hospital resources. The aim of this study is to investigate the association between Sequential Organ Failure Assessment (SOFA) scale scores and mortality of patients admitted to an intensive care unit. This is a prospective cohort study with document analysis. The respiratory and coagulation systems were
\end{abstract}


not associated with mortality. However, the scores of the other 4 systems (hepatic, cardiovascular, neurological and renal) showed a strong association with mortality in the study sample (p-value <0.05). It was possible to show that the SOFA scale is a predictor of mortality associated with organ dysfunction in patients with infection, hospitalized in a intensive care unit.

Keywords: Sepsis; Organ dysfunction scores; SOFA scale; Intensive care units.

\section{Resumen}

La sepsis es una síndrome que se puede definir como una respuesta sistémica y desordenada a una patología infecciosa específica, provocada por cualquier microorganismo, que se presenta en diferentes estadios clínicos de un mismo proceso fisiopatológico. La identificación precoz de la sepsis es fundamental, además del uso de escalas para identificar rápidamente los órganos afectados, con el fin de optimizar la administración de determinados recursos hospitalarios. El objetivo de este estudio es investigar la asociación entre laspuntuaciones de la escala Sequential Organ Failure Assessmen (SOFA) y la mortalidad en pacientes ingresados en una unidad de cuidados intensivos. Este es un estudio de cohorte prospectivo con análisis de documentos. Lo sistema respiratorioy de coagulación no se asociaron con la mortalidad. Sin embargo, las puntuaciones de los otros 4 sistemas (hepático, cardiovascular, neurológico y renal) mostraron una fuerte asociación con la mortalidad en la muestra de estudio (valor de $\mathrm{p}<0,05$ ). Se pudo demostrar que la escala SOFA es unpredictor de mortalidad asociada a disfunción orgánica en pacientes hospitalizados por infección, en una unidade de cuidados intensivos.

Palabras clave: Sepsis; Puntuaciones en la disfunción de órganos; Puntuaciones SOFAS; Unidades de cuidados intensivos.

\section{Introdução}

As unidades de terapia intensiva (UTI) são áreas críticas destinadas à internação de pacientes graves, que apresentam comprometimento de um ou mais dos principais sistemas fisiológicos, com perda de sua autorregulação, necessitando de assistência contínua, de profissionais especializados, materiais específicos e tecnologias necessárias ao diagnóstico, monitorização e terapia (Chowdhury \& Duggal, 2017; Silva et al, 2020).

Tão importante quanto à aplicação de recursos, novos tratamentos e tecnologias de ponta nas UTI, é a avaliação adequada dos pacientes que apresentam alto risco de infecções relacionadas ao cuidado em saúde devido ao grande número de dispositivos invasivos, como drenos, cateteres ou tubo orotraqueal (Brasil, Agência Nacional de Vigilância Sanitária [ANVISA], 2010). As infecções são um quadro frequente nos pacientes internados nas UTI, muitas vezes evoluindo para uma situação de acometimento dos órgãos (sepse), acarretando em alto índice de mortalidade (Junior \& da Silva, 2014; de Freitas et al, 2014).

A sepse é uma síndrome definida como uma resposta sistêmica e desordenada a determinado quadro infeccioso, causado por qualquer microrganismo, apresentando-se em diferentes estágios clínicos de um mesmo processo fisiopatológico. Os dados epidemiológicos disponíveis apontam a sepse como a causa principal de mortes nas UTI e, neste sentido, é fundamental sua identificação precoce além da utilização de escalas para identificar rapidamente os órgãos acometidos, para otimizar a administração de determinados recursos hospitalares e a aumentar qualidade no cuidado (Borges et al, 2020; Instituto Latino-americano de Sepse [ILAS], 2015).

O Sequential Organ Failure Assessment (SOFA) é utilizado atualmente para quantificar diariamente o grau da disfunção dos órgãos nos pacientes, que é a característica mais determinante para o diagnóstico, tratamento e custo hospitalar. Nesta escala, cada sistema (respiratório, hematológico, hepático, cardiovascular, neurológico e renal) é avaliado a partir de variáveis objetivas, disponíveis nas rotinas das UTI e escores mais altos estão associados com aumento na probabilidade de mortalidade (Seymour et al, 2016; van Vught et al, 2016; Carvalho et al, 2021). O objetivo deste estudo é investigar a associação entre os escores da escala de SOFA e a mortalidade de pacientes internados em uma UTI.

\section{Metodologia}

Trata-se de um estudo de coorte prospectivo, com análise documental (Hulley et al, 2015). Os participantes foram 
recrutados na unidade de terapia intensiva de um hospital público situado no município do Rio de Janeiro, RJ, Brasil. A população consistiu de pessoas adultas, hospitalizadas em 10 leitos de internação, sendo utilizada uma amostra por conveniência, formada por uma coorte aberta dos pacientes internados no período de 19 de março a 19 de maio de 2018.

Foram usados como critérios de inclusão: ter idade maior que 18 anos, permanência de, no mínimo, 24 horas na UTI, apresentar quadro de infecção e estar sob ventilação mecânica. Foram excluídos pacientes com morte encefálica confirmada nas primeiras 24 horas de internação. Os participantes foram acompanhados até o momento da alta para a residência ou ocorrência de óbito intra-hospitalar.

Com base na média de pacientes internados nos seis últimos meses de 2017, foi estimado o tamanho amostral de 32 pacientes para se obter um nível de confiança de $95 \%$. A coleta de dados foi conduzida por um enfermeiro preceptor e duas estudantes de enfermagem (adequadamente treinadas), que se organizaram em uma escala diária, durante dois meses, e as informações foram registradas em um instrumento desenvolvido pelos pesquisadores, contendo variáveis sociodemográficas e aspectos clínicos dos pacientes.

A partir dos dados coletados, foi calculado diariamente o escore de SOFA para cada participante. O desfecho principal foi a mortalidade intra-hospitalar e as variáveis independentes foram: idade média, sexo, valor médio do SOFA e valor médio de SOFA para cada sistema (respiratório, hematológico, hepático, cardiovascular, neurológico e renal).

Os dados foram analisados através do software Epi Info 7.2, do Centers for Disease Control and Prevention (CDC). A análise foi feita através de estatística descritiva e, adicionalmente, por regressão. Um valor de significância inferior a 5\% (p $<0,05)$ foi considerado estatisticamente significativo. Este estudo é um recorte do projeto intitulado "A prevenção de pneumonia associada à ventilação mecânica em UTI", submetido para apreciação ética e aprovação através da Plataforma Brasil, sob o número de protocolo CAAE: 71190317.6.0000.5291.

\section{Resultados e Discussão}

A distribuição dos participantes segundo sexo e desfechos clínicosé apresentada na Tabela 1, sendo a amostra final composta por 40 participantes: 19 homens e 21 mulheres. Durante o período de seguimento, 16 participantes (40\%) evoluíram para o óbito na UTI, 24 participantes (60\%) tiveram alta para outro setor, e destes últimos apenas um $(2,5 \%)$ evoluiu para óbito antes da alta para a residência.

Tabela 1 - Distribuição dos participantes segundo sexo e desfecho clínico - Rio de Janeiro - 2021.

\begin{tabular}{lrrr}
\hline & Característica & $\mathrm{N}$ & $(\%)$ \\
\hline Sexo & Feminino & 21 & 52,50 \\
& Masculino & 19 & 47,50 \\
Motivo da alta da UTI & & & \\
& Melhora do quadro & 24 & 60,00 \\
Motivo da alta hospitalar & Óbito & 16 & 40,00 \\
& & & 57,50 \\
& Melhora do quadro & 23 & 17 \\
\hline
\end{tabular}

Fonte: Elaborado pelos autores.

A idade média dos participantes foi de 47,78 anos, havendo uma pequena diferença entre os gêneros, ficando o feminino com uma média de 52,73 anos. O tempo médio de internação hospitalar foi de 33,92 dias, já o tempo médio de internação na UTI foi de 17,32 dias. 
A Tabela 2 apresenta a caracterização da amostra estratificada em relação ao sexo dos participantes da amostra, destacando a grande diferença entre os tempos de internação na UTI entre homens e mulheres, tendo os primeiros um tempo mais de $100 \%$ maior. Isto pode ter como causa provável o grande índice de participantes do sexo masculino internados por causas violentas, o que resulta em quadros mais severos e consequente aumento no tempo de internação na UTI (Dyusembekov, et al, 2018).

Tabela 2 - Idade média, tempo de internação na UTI e tempo de internação hospitalar, estratificados por sexo - Rio de Janeiro - 2021.

\begin{tabular}{|c|c|c|c|c|}
\hline & Característica & $\mathrm{N}$ & Média & Desvio Padrão \\
\hline \multicolumn{5}{|l|}{ Idade (anos) } \\
\hline & Feminino & 21 & 52,73 & 16,79 \\
\hline & Masculino & 19 & 41,07 & 19,05 \\
\hline & Geral & 40 & 47,78 & 18,92 \\
\hline \multicolumn{5}{|l|}{ Tempo de internação na UTI (dias) } \\
\hline & Feminino & 21 & 11,90 & 16,51 \\
\hline & Masculino & 19 & 23,31 & 18,51 \\
\hline & Geral & 40 & 17,32 & 18,20 \\
\hline \multicolumn{5}{|l|}{ Tempo de internação hospitalar (dias) } \\
\hline & Feminino & 21 & 31,42 & 23,20 \\
\hline & Masculino & 19 & 36,68 & 21,55 \\
\hline & Geral & 40 & 33,92 & 22,31 \\
\hline
\end{tabular}

Fonte: Elaborado pelos autores.

As principais comorbidades associadas aos participantes são apresentadas na Tabela 3, sendo as principais a hipertensão arterial e o diabetes mellitus. Os registros da equipe multiprofissional tiveram escassez de informações sobre as comorbidades e apenas 12 participantes possuíam estas informações.

Tabela 3 - Comorbidades associadas aos participantes - Rio de Janeiro - 2021.

\begin{tabular}{ccc}
\hline Comorbidades & $\mathrm{N}$ & $\%$ \\
\hline Hipertensão arterial & 6 & 50,00 \\
Diabetes mellitus & 2 & 25,00 \\
Doença pulmonar obstrutiva crônica & 1 & 6,25 \\
Imunossupressão & 1 & 6,25 \\
Insuficiência renal & 1 & 6,25 \\
Arritmia & 1 & 6,25 \\
\hline Total & 12 & 100 \\
\hline
\end{tabular}

Fonte: Elaborado pelos autores.

Durante a coleta de dados, foi identificada ausência de informações para o cálculo adequado do escore de SOFA, conforme apresentado na Tabela 4. Os sistemas respiratório e hepático foram os mais afetados, devido à irregularidade na presença de resultados diários de gasometria arterial e mensuração de bilirrubina. De acordo com a definição da escala de SOFA, os sistemas não avaliados por falta de informação recebem a pontuação zero, impactando o escore total (Raithet al, 2017; Pinto, Santos \& Simor, 2021). 
Tabela 4 - Ausência de avaliação de sistemas no cálculo do Escore de SOFA - Rio de Janeiro - 2021.

\begin{tabular}{rrcc}
\hline Sistema & $\mathrm{N}$ & $\%$ & 40,28 \\
& Respiratório & 226 & 14,16 \\
Coagulação & 82 & 34,58 \\
Hepático & 194 & 3,2 \\
Cardiovascular & 18 & 13,37 \\
Nervoso Central & 74 & 15,33 \\
\hline
\end{tabular}

Fonte: Elaborado pelos autores.

As comparações entre os valores parciais do SOFA para os seis sistemas independentes são apresentadas na Tabela 5. As pontuações da escala de SOFA para o sistema respiratório e para o de coagulação não apresentaram associação com a mortalidade Entretanto, as pontuações de SOFA para os outros 4 sistemas apresentaram forte associação com a mortalidade na amostra de estudo (p-valor <0,05).

Tabela 5 - Mortalidade entre os participantes por valor médio do escore de SOFA e por pontuações médias dos sistemas - Rio de Janeiro - 2021.

\begin{tabular}{rccc}
\hline Variável & Sobreviventes & Não sobreviventes & $\begin{array}{c}\text { P-valor (sobreviventes versus não } \\
\text { sobreviventes) }\end{array}$ \\
\hline Escore de SOFA & 3,43 & 4,52 & 0,018 \\
Respiração & 0,24 & 0,43 & 0,817 \\
Coagulação & 0,15 & 0,34 & 0,442 \\
Hepático & 0,10 & 0,49 & 0,011 \\
Cardiovascular & 1,04 & 2,09 & 0,009 \\
Sistema Nervoso Central & 0,45 & 1,34 & $<0,001$ \\
Renal & 0,65 & 0,91 & $<0,001$ \\
\hline
\end{tabular}

Fonte: Elaborado pelos autores.

A Figura 1 apresenta os diagramas de Pareto para os valores médios do escores de SOFA para a amostra estudada. Entre o grupo de não sobreviventes as maiores contribuição ocorreram para valores médios do escore de SOFA entre 3 e 5. Para o grupo de sobreviventes as maiores contribuições foram devidas ao valor médio do escore de SOFA igual a 1. 
Figura 1 - diagramas de Pareto para os valores médios do escores de SOFA diagramas de Pareto para os valores médios do escores de SOFA - Rio de Janeiro - 2021.
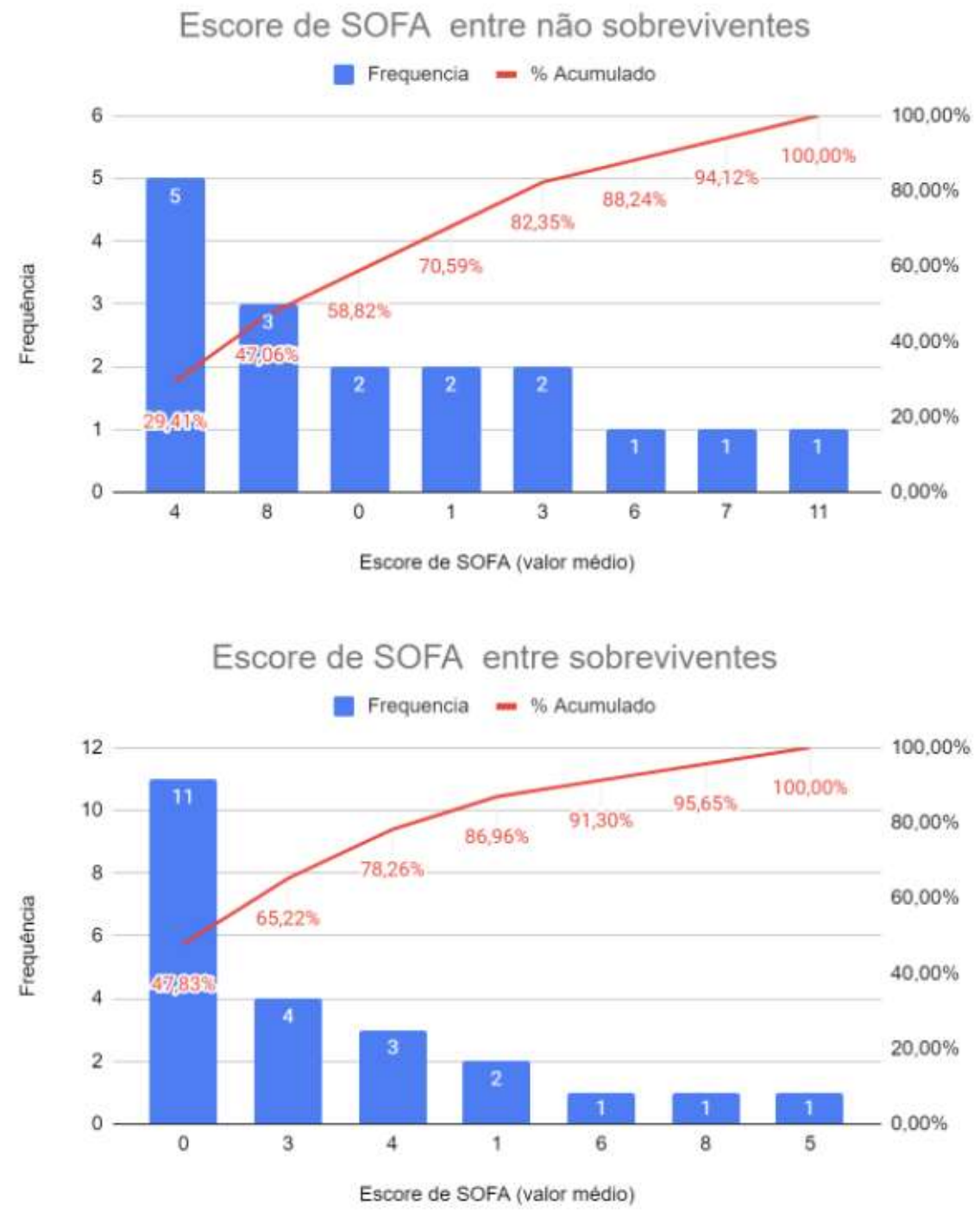

Fonte: Elaborado pelos autores.

\section{Conclusão}

Os dados obtidos e a análise estatística deste estudo, sugerem que a escala de SOFA pode ser utilizada como um instrumento preditor da mortalidade associada à disfunção orgânica em pacientes internados em uma UTI, já que tanto o escore de SOFA médio, quanto as pontuações médias para os sistemas apresentaram forte correlação com a mortalidade dos participantes. O estudo apresentou como principais fragilidades a grande ausência de informações sobre resultados laboratoriais para o cálculo do escore de SOFA.

Torna-se importante a realização de outros estudos brasileiros, principalmente multicêntricos, com amostras maiores e cenários diversos, a fim de serem obtidos resuldados mais contundentes para a determinação de políticas públicas para prevenção, identificação precoce e tratamento da sepse. 


\section{Referências}

Borges, A. C. do N., Costa, A. L., Bezerra, J. B., Araújo, D. S., Soares, M. A. A., Gonçalves, J. N. de A., Rodrigues, D. T. da S., Oliveira, E. H. S. De, Luz, L. E. Da, Silva, T. R., \& Silva, L. G. de S. (2020). Epidemiology and pathophysiology of sepsis: an review. Research, Society and Development, 9(2), e187922112.https://doi.org/10.33448/rsd-v9i2.2112

Brasil. Agência Nacional de Vigilância Sanitária (2010). Dispõe sobre os requisitos mínimos para funcionamento de Unidades de Terapia Intensiva e dá outras providências. https://bvsms.saude.gov.br/bvs/saudelegis/anvisa/2010/res0007_24_02_2010.html

Carvalho, P. P., Porto, M. de J., Barros, J. F., Magalhães, A. S., Nunes, L. S., Silva, I. C., Abreu, I. L. S. de, Souza, G. N., Rezende Neto, J. M. de., \& Mendes, T. da S. (2021). Evaluation of critical patients with suspected sepsis in a university hospital. Research, Society and Development, 10(3), e39410313570. https://doi.org/10.33448/rsd-v10i3.13570

Chowdhury, D., \& Duggal A. K. (2017). Intensive care unit models: Do you want them to be open or closed? A criticalreview. NeurolIndia, 65(1), 39-45. 10.4103/0028-3886.198205. PMID: 28084236.

de Freitas, G. R. C., Da Fonseca-Neto, O. C. L., Pinheiro, C. L. F., Araújo, L. C., Barbosa, R. E. N., \& Alves, P. (2014). Relação entre o Sequential Organ Failure Assessment (SOFA) e a pressão intra-abdominal em unidade de tratamento intensivo. Arq Bras Cir Digl. 27(4). http://www.scielo.br/scielo.php?pid=S010267202014000400256\&script=sci_arttext\&tlng=pt

Dyusembekov, E., Akhanov, G., Aliev, M., Uteuliyev, Y., \& Saktapov, A. (2018). Georgian medical news, (285), 17-20.

Hulley, S. B., Cummings, S. R., Browner, W. S., \& Grady, D. G. (2014). Delineando a pesquisa, Porto Alegre, Artmed.

Instituto Americano de Sepse. (2015). Sepse: um problema de saúde pública. Brasília: CFM.

Júnior, L. C. M. C., \& Da Silva, R. R. (2014). Sepse em pacientes internados em unidade de terapia intensiva com lesão cerebral traumática: fatores associados a maior mortalidade. Rev Bras Ter Intensiva, 26 (2): 148-154. http://www.rbti.org.br/artigo/detalhes/0103507X-26-2-11

Pinto, C. da S. P., Santos, M. V. dos., \& Simor, A. (2021). Control factors and progression of sepsis in the Intensive Care Unit: a literature review. Research, Society and Development, 10(14), e38101421750. https://doi.org/10.33448/rsd-v10i14.21750

Raith, E. P., Udy, A. A., Bailey, M., McGloughlin, S., MacIsaac, C., Bellomo, R., Pilcher, D. V., \& Australian and New Zealand Intensive Care Society (ANZICS) Centre for Outcomes and Resource Evaluation (CORE) (2017). Prognostic Accuracy of the SOFA Score, SIRS Criteria, and qSOFA Score for InHospital Mortality Among Adults With Suspected Infection Admitted to the Intensive Care Unit. JAMA, 317(3), 290-300. https://doi.org/10.1001/jama.2016.20328

Seymour, C. W., Liu, V. X., Iwashyna, T. J., Brunkhorst, F. M., Rea, T. D., \& Scherag, A. (2016). Assessment of Clinical Criteria for Sepsis: For the Third International Consensus Definitions for Sepsis and Septic Shock (Sepsis-3). Rev JAMA. 315(8): 762-74. https://jamanetwork.com/journals/jama/fullarticle/2492875

Silva, L. E. C., Lima, M. B. e S., Silva \& Államy, D. M. E. (2020). Nurse's performance in the prevention and identification of sepsis signs and symptoms in intensive therapy: an integrative review. Research, Society and Development, 9(11), e1229119599. https://doi.org/10.33448/rsd-v9i11.9599

van Vught, L. A., Klouwenberg, P. M. K., Spitoni, C., Scicluna, B. P., Wiewel, M. A., Horn, J., Schultz, M. J., Nürnberg, P., Bonten, M. J., Cremer, O. L., \& van der Poll, T. (2016), MARS Consortium. Incidence, Risk Factors, and Attributable Mortality of Secondary Infections in the Intensive Care Unit After Admission for Sepsis. JAMA. 12;315(14):1469-79. 10.1001/jama.2016.2691. PMID: 26975785 